\title{
Multi-scale Order Recurrence Plot based deterministic analysis on Heart Rate Variability in Congestive Heart Failure Assessment
}

\author{
Hu Yuhang ${ }^{1 *}$ \\ ${ }^{1}$ Software engineering, School of Information and Communications Technology, Xiamen University Malaysia, Malaysia
}

\begin{abstract}
Congestive heart failure (CHF) is a cardiovascular disease associated with the abnormal autonomic nervous system (ANS). Heart rate variability analysis (HRV) is the main method for the quantitative evaluation of autonomic nervous function. Common analytical methods of HRV include time domain, frequency domain, and nonlinear methods. However, these methods generally ignore the short-term volatility of heart rate and autonomic ganglion law. Therefore, this study proposes a new parameter to analyze heart rate variability-determination of a multi-scale order recurrence plot (MSORP_DET). This method can analyze the HRV in patients with heart failure on multiple time scales. This study analyzed the R-R interval in 24-hour HRV data from 98 samples (54 normal subjects and 44 patients with CHF). The results showed that MSORP_DET could significantly distinguish CHF patients from normal subjects $(p<0.001)$. Moreover, the accuracy rate of screening patients with CHF reached the maximum of $81.6 \%$ by using the combination of low frequency/high frequency (LF/HF) and MSORP_DET, compared with $78.6 \%$ when using LF/HF alone. Therefore, MSORP_DET can be used as a new index to screen patients with CHF and reveal that the rhythm of ANS in patients with heart failure is more complex than that in normal people.
\end{abstract}

\section{Introduction}

Congestive heart failure (CHF) is a common clinical disease that leads to pulmonary vascular congestion and reduced cardiac output [1]. The morbidity and mortality of this disease are high [2]. Many forms of heart disease can lead to congestive heart failure [2]. And patients with CHF are prone to other complications, including obstructive sleep apnea, pulmonary edema, and pleural effusion [1]. However, there are no early symptoms in patients with CHF [3]. Therefore, rapid and accurate detection of CHF has important clinical significance.

Studies have shown that CHF is associated with abnormalities of ANS. The increase of sympathetic nerve activity increases the contractility of the heart and shortens the systolic period, which leads to the increase of heart rate, and the parasympathetic nerve affects the sinoatrial node and decreases the heart rate. Under normal physiological conditions, sympathetic and parasympathetic nerves coordinate the heart's rhythm changes through complex interactions. Once this interaction is out of balance due to diseases and other reasons, it can lead to cardiovascular dysfunction and changes in heart rate variability.

Analysis of heart rate variability (HRV) is a reliable and reproducible technique for analyzing and evaluating autonomic activity in patients with CHF [4]. Previous studies have shown that the classical HRV indices of patients with CHF are significantly different from those of normal people [5]. Traditionally, frequency domain HRV indices are commonly used for analyzing HRV.
Pagani et al. proposed that the ratio of LF to HF (LF/HF) could quantify the changing relationship between sympathetic and parasympathetic nerve activities [6], $\mathrm{LF} / \mathrm{HF}$ has been widely accepted as a tool for assessing cardiovascular autonomic regulation. The changes in the continuous cardiac cycle (R-R interval) are divided into different frequency components, usually identifying two peaks: high frequency (HF), 0.15-0.4 HZ, and low frequency (LF), 0.04-0.15 HZ. An increase in the LF/HF index is assumed to reflect a shift to "sympathetic dominance", and a decrease in the index corresponding to a "parasympathetic dominance". Studies have shown that the LF/HF of patients with CHF is significantly lower than normal people. However, George demonstrated that LF/HF cannot accurately measure sympathetic balance, since the HF power cannot be solely attributed to changes in cardiac parasympathetic nerves. And the selection of only high frequency and low frequency greatly simplifies the complex nonlinear interaction between sympathetic and parasympathetic nerves in the autonomic nervous system [6]. Moreover, LF/HF can only evaluate the overall level of the HRV but ignore the short-term volatility of heart rate and autonomic ganglion law. The length of time segments to calculate the LF/HF is usually 1 minute or 5 minutes [7]. And the LF/HF are linear indicators, while autonomic nervous systems are nonlinear.

To solve these problems, Europhys et al. proposed the recurrence plot based on state space reconstruction, which can reveal the internal structure of time series and obtain information about similarity and complexity [8]. The

\footnotetext{
* Corresponding author: SWE1809351@xmu.edu.my
} 
order recurrence plot is a new method of time series analysis. It describes a sequence as a series of sort patterns according to a particular sequence of events. Multi-scale order recurrence plot (MSORP) investigates the nonlinear dynamic characteristics of signals at multiple time scales by introducing different time scales. The recursive quantitative analysis (RQA) proposed by Zbilut et al. can quantify the recurrence plot and summarize the information in a set of indicators or statistics. RQA has become a new nonlinear time series analysis tool that does not need to consider the low-dimensional chaotic characteristics of signals. Also, it is particularly useful in physiological signal analysis because stationary longterm physiological signal sequences are difficult to obtain [9]. The analytical indexes introduced by the RQA are DIV (reciprocal of the longest diagonal line), DET (the percentage of the recursive points forming the diagonal line segment to the total recursive point), the ENT (Shannon entropy or the measure of the average information contained in the line segment distribution), etc. Within the RQA framework, the basic characteristic of the existence of structures (linear or nonlinear) is the existence of lines parallel to the principal diagonal [10]. Among the above three indicators, DET most clearly indicates this feature. Thus, in this study, DET is chosen to analyze the dynamic characteristics of heart rate fluctuation.

Converting one-dimensional physiological signals into a multi-scale order recurrence plot and calculating its MSORP_DET index can better analyze the nonlinear dynamic characteristics. This study extracted 5-minute R$\mathrm{R}$ fragments from HRV signals of normal subjects and patients with $\mathrm{CHF}$ and then calculated $\mathrm{LF} / \mathrm{HF}$ and MSORP_DET. After that, the Fisher discriminant function is used to test whether the two indices can correctly distinguish whether each sample is a normal or CHF patient.

\section{Methods}

\subsection{Data}

In this study, the electrocardiogram (ECG) recordings were derived from the open database of the Physionet website. The 24-h RR interval signals for 54 healthy subjects (age: 61.38 \pm 11.63 years, 31 males and 23 females) were collected from the Normal Sinus Rhythms RR Interval database, and $44 \mathrm{CHF}$ subjects (age: $55.51 \pm 11.44$ years, 19 males and six females, 19 subjects' gender were unknown) consisted of 29 from the Congestive Heart Failure RR interval database and 15 from the Beth Israel Deaconess Medical Center (BIDMC) Congestive Heart Failure Database. In later $\mathrm{CHF}$ assessment, the $44 \mathrm{CHF}$ subjects were categorized into the CHF group, and the 54 healthy subjects were categorized into the control group. In this study, the HRV features of the control group and the CHF group would be analyzed by using LF/HF and MSORP_DET. The framework of the analysis system is shown in Figure 1.

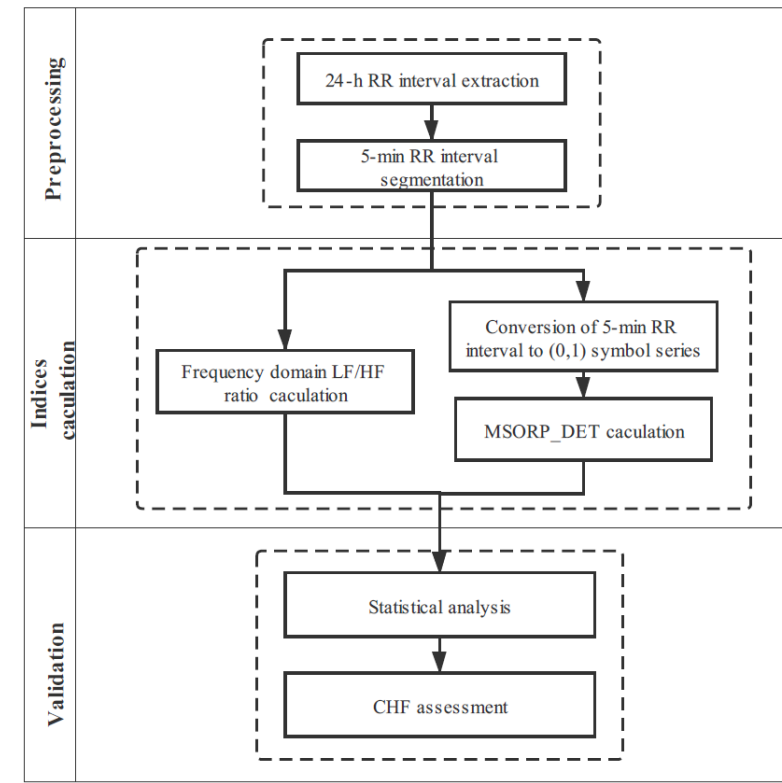

Fig. 1. Framework of the proposed HRV analysis system.

First, 24-h RR interval recordings were extracted from the electrocardiogram and then segmented into 5-min nonoverlapping epochs. Then, the frequency domain LF/HF ratio and MSORP DET were calculated based on the 5-min epochs. To calculate MSORP DET, the 5-min epochs were converted to $(0,1)$ symbol series first. Finally, these two indices were validated by statistical analysis and CHF assessments.

In the data preprocessing stage of this study, the method of binary symbolizations of the RR interval series proposed by Cysarz et al. was used. The succession of accelerations and decelerations of the RR interval was expressed by 1 and 0 , respectively [11], which corresponding to sympathetic and parasympathetic excitations. This method can capture large-scale features and reduce the impact of measurement noise, thus improving the accuracy of the experiment.

\subsection{Multi-scale Order Recurrence Plot (MSORP) and Recurrence Quantification Analysis (RQA)}

\section{Multi-scale measures}

Given an original time series $\left\{\mathrm{x}_{1}, \mathrm{x}_{2}, \ldots, \mathrm{x}_{\mathrm{N}}\right\}$, coarsegraining the time series by averaging the time data points within non-overlapping time slice at a time scale. Then the time series is converted into a successive coarsegrained version. Each element of the coarse-grained time series $\left\{\mathrm{y}^{(\mathrm{s})}\right\}$ at time scale $s$ is constructed by

$$
\mathrm{y}_{\mathrm{j}}^{(\mathrm{s})}=\frac{1}{\mathrm{~s}} \sum_{\mathrm{i}=\mathrm{j}}^{\mathrm{j}+\mathrm{s}-1} \mathrm{x}_{\mathrm{i}}, 1 \leq \mathrm{j} \leq \mathrm{N}-\mathrm{s}+1
$$

\section{Order recurrence plot}

Given the embedding dimension $\mathrm{m}$ and delay time $\tau$, the original phase space of the coarse-grained time series is reconstructed as $\mathrm{m}$-dimensional phase space. The time series in the m-dimensional phase space can be expressed as:

$$
X_{k}=\left(x_{k}, X_{k+\tau}, \ldots, X_{k+(m-1) \tau}\right), k=1,2, \ldots, L-(m-1) \tau
$$


Then $\mathrm{X}_{\mathrm{k}}$ is arranged in an increasing order:

$$
\mathrm{x}_{\mathrm{k}+\left(\mathrm{t}_{1}-1\right) \tau} \leq \mathrm{x}_{\mathrm{k}+\left(\mathrm{t}_{2}-1\right) \tau} \leq \ldots \leq \mathrm{x}_{\mathrm{k}+\left(\mathrm{t}_{\mathrm{m}}-1\right) \tau}
$$

If $x_{k+\left(t_{1}-1\right) \tau}=x_{k+\left(t_{2}-1\right) \tau}$, the values are ordered as $x_{k+\left(t_{1}-1\right) \tau} \leq x_{k+\left(t_{2}-1\right) \tau}$ for $t_{1}<t_{2}$. The order pattern is denoted as $\pi$. For example, given a time series $X=\{2,4,3\}, m=3$, its order pattern is $\pi=\{0,1,2\}$ because $\mathrm{x}_{1}<\mathrm{x}_{2}<\mathrm{X}_{3}$.

After getting the order pattern $\pi$, for any two order patterns $\pi_{\mathrm{i}}$ and $\pi_{\mathrm{j}}$ of states $\mathrm{i}$ and $\mathrm{j}$, we define the order patterns recurrence plot as:

$$
R_{i j}=\left\{\begin{array}{l}
0: \pi_{i} \neq \pi_{j} \\
1: \pi_{i}=\pi_{j}
\end{array} \quad(i, j=1,2, \ldots, N)\right.
$$

We get a recurrence matrix of the points within the mdimensional space. If the states of $\mathrm{i}$ and $\mathrm{j}$ are the same, $\mathrm{R}_{\mathrm{ij}}$ $=1$, otherwise $R_{i j}=0$. The recurrence matrix determines the existence of recursion in any two spatial states by comparing their order patterns.

\section{Determinism}

To quantify the characteristics of the order recurrence plot, determinism (DET) is used to measure its complexity. DET is defined as the proportion of the recurrence plots on the diagonal structures to all recurrence plots. Its formula is shown below:

$$
\mathrm{DET}=\sum_{\mathrm{l}=\mathrm{l}_{\min }}^{\mathrm{N}} \mathrm{IP}(\mathrm{l}) / \sum_{\mathrm{l}=1}^{\mathrm{N}} \mathrm{IP}(\mathrm{l})
$$

$\mathrm{P}(1)$ is the total number of the 1 length diagonal structures in the recurrence plot. The $l_{\min }$ is the minimum length of the diagonal line, and only the diagonal line with a length greater than or equal to lmin is counted. When $\operatorname{lmin}=1$, DET $=1$.

In this study, the threshold value lmin was set to $3,4,5$ to select the optimal one with the highest accuracy. The embedding dimension $\mathrm{m}$ is set to 3 and $\tau$ is set to 1 . And the multiple time scale $\mathrm{S}$ was set between a wide range of 15 and 25, with a total of 11 values. Figure 2. shows the calculation process of MSORP_DET.

Firstly, coarse-graining the time series with the scale factor S. Secondly, reconstruct the time series into $\mathrm{m}$ dimension with delay vector $\tau$. Then sort the time series in ascending order. Finally, calculate the DET index of the new time series.

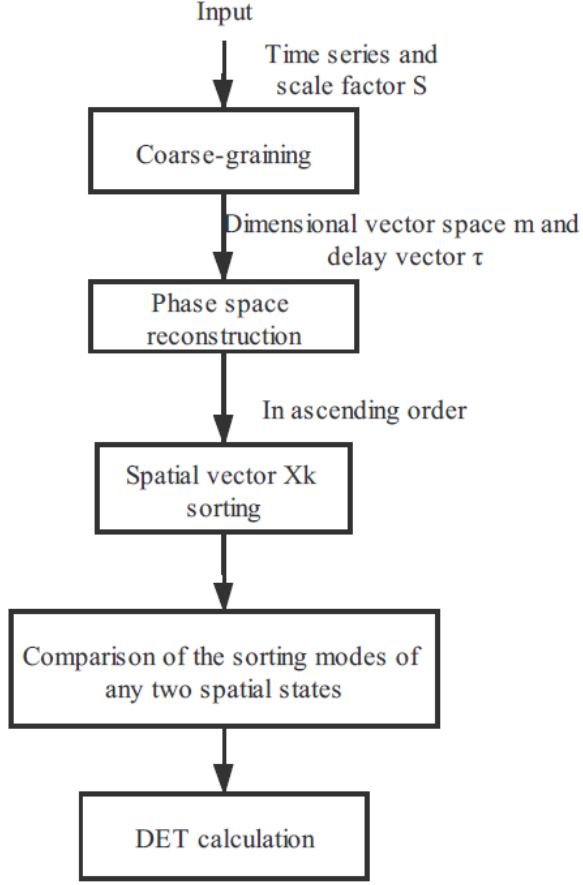

Fig. 2. The flow chart of MSORP_DET method.

\subsection{LF/HF ratio}

The fast Fourier transform was used to calculate the power spectral density of the RR intervals. LF is the component of frequency integral range $0.04-0.15 \mathrm{~Hz}, \mathrm{HF}$ is the component of frequency integral range $0.15-0.4 \mathrm{~Hz}$ [12]. The formula of LF/HF ratio is shown below:

$$
\mathrm{LF} / \mathrm{HF} \text { ratio }=\frac{\mathrm{LF} \text { power }}{\mathrm{HF} \text { power }}
$$

\subsection{Statistical Analysis}

First, the significant difference between the control group and CHF group was analyzed by an independent sample t-test, $\mathrm{p}<0.05$ was considered statistically significant. Further, for CHF assessment, Fisher's discriminant function was used to classify each sample as normal or CHF and obtain the accuracy, specificity, and sensitivity parameters of LF/HF and MSORP_DET. These tests were performed using SPSS version 22.0.0.0 (SPSS Inc., Chicago, IL, USA).

\section{Results}

\subsection{MSORP_DET can distinguish between the control and $\bar{C}$ HF groups.}

The mean value and standard deviation of $\mathrm{LF} / \mathrm{HF}$ ratio and MSORP DET for the control and CHF groups were shown in Table 1 . The mean values of these two indices of the CHF group are smaller than those of the control group. The $p$ values of LF/HF ratio and MSORP_DET were less than 0.001 , indicating that both $\mathrm{LF} / \mathrm{HF}$ ratio and MSORP_DET can show a significant difference between the control and CHF groups. Figure 3. shows the results of LF/HF ratio and MSORP_DET index in the control and 
CHF groups. It can be seen that these two indices of the CHF group are smaller than those of the control group.

Table 1. Values of LF/HF and MSORP_DET indices for CHF and Control group.

\begin{tabular}{|c|c|c|}
\hline Indices & LF/HF & MSORP_DET \\
\hline $\begin{array}{c}\text { Normal group } \\
\text { (mean } \pm \text { SD) }\end{array}$ & $3.3684 \pm 1.02232$ & $0.4335 \pm 0.034$ \\
\hline $\begin{array}{c}\text { CHF group } \\
\text { (mean } \pm \text { SD) }\end{array}$ & $1.7703 \pm 1.19596$ & $0.4036 \pm 0.02349$ \\
\hline p-value & $0.000^{* * *}$ & $0.000^{* * *}$ \\
\hline
\end{tabular}

SD: standard deviation; $* * *$ represents $\mathrm{p}<0.001$ between Control and CHF groups.

$\mathbf{A}$

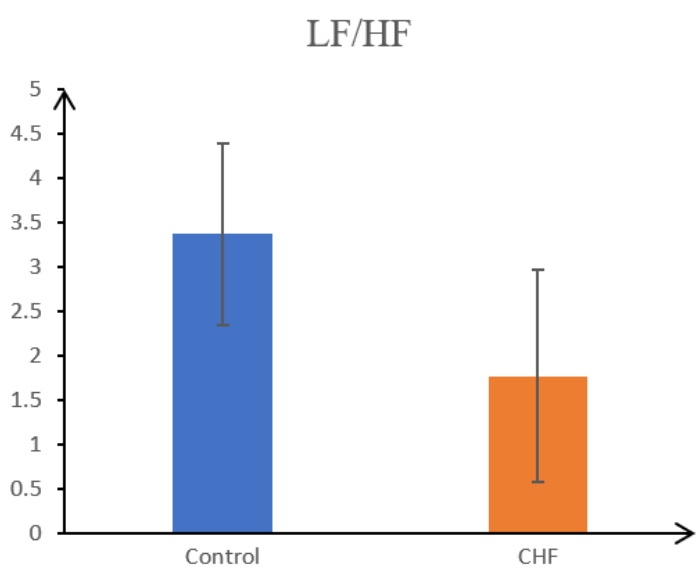

B

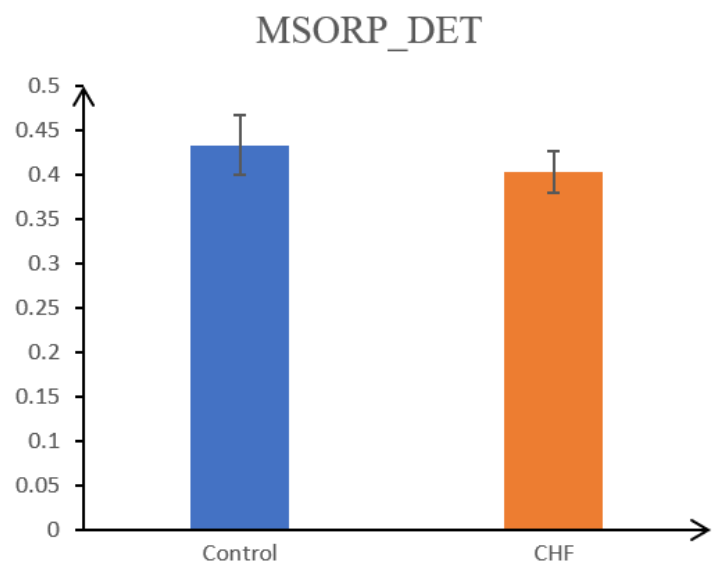

Fig. 3. The LF/HF ratio of the control and CHF groups (A). The MSORP_DET of the control and CHF groups (B). *** represents $\mathrm{p}<0.001$.

\subsection{The combination of LF/HF and MSORP_DET achieved the highest accuracy in CHF assessment.}

CHF assessment was used to validate the performance of LF/HF, MSORP_DET and LF/HF+MSORP_DET. The Fisher's discriminant function was carried out to classify each sample as a healthy or CHF subject. Table2 showed the accuracy (Acc), sensitivity (Sen), and specificity (Spe) of these three indicators in screening patients with congestive heart failure. The accuracy represents the proportion of accurately classified samples; the sensitivity represents the proportion of accurately classified $\mathrm{CHF}$ subjects; and the specificity represents the proportion of accurately classified health subjects. As shown in Table 2, the Acc of the MSORP_DET was $69.4 \%$, Sen was $77.3 \%$, and Spe was $63 \%$; the Acc of the LF/HF was $78.6 \%$, Sen was $81.8 \%$ and Spe was $75.9 \%$. Although the Acc, Sen and Spe values of MSORP DET were lower than those of the LF/HF index, the Acc was obviously improved to $81.6 \%$ by combining the MSORP DET and LF/HF indices, and the Sen remained at $81.8 \%$, the Spe increased to $81.5 \%$. It can be seen that the combination of the two indices can improve the screening effect than using separate indices.

Table 2. Performance of indices for CHF screening.

\begin{tabular}{|c|c|c|c|}
\hline Indices & Acc/\% & Sen/\% & Spe/\% \\
\hline LF/HF & 78.6 & 81.8 & 75.9 \\
\hline MSORP_DET & 69.4 & 77.3 & 63 \\
\hline LF/HF and MSORP_DET & 81.6 & 81.8 & 81.5 \\
\hline
\end{tabular}

Acc: accuracy; Sen: sensitivity; Spe: specificity

\subsection{The accuracy reached a maximum of $69.4 \%$ when $I \min =4$ and $S=19$}

Figure 4 shows the different accuracy corresponding to different scale factor $\mathrm{S}$ and diagonal length $1 \mathrm{~min}$. It can be seen that the fluctuation direction of three curves with $\operatorname{lmin}=3,4,5$ is generally the same, and the curves fluctuate obviously with the change of $\mathrm{S}$ value. This shows that accuracy is greatly influenced by scale factor $\mathrm{S}$ and less by $\operatorname{lmin}$. When $\operatorname{lmin}=4, S=19$, the accuracy reached a maximum of $69.4 \%$. Therefore, this study chose the MSORP_DET with $\operatorname{lmin}=4, \mathrm{~S}=19$ to combine with $\mathrm{LF} / \mathrm{HF}$.

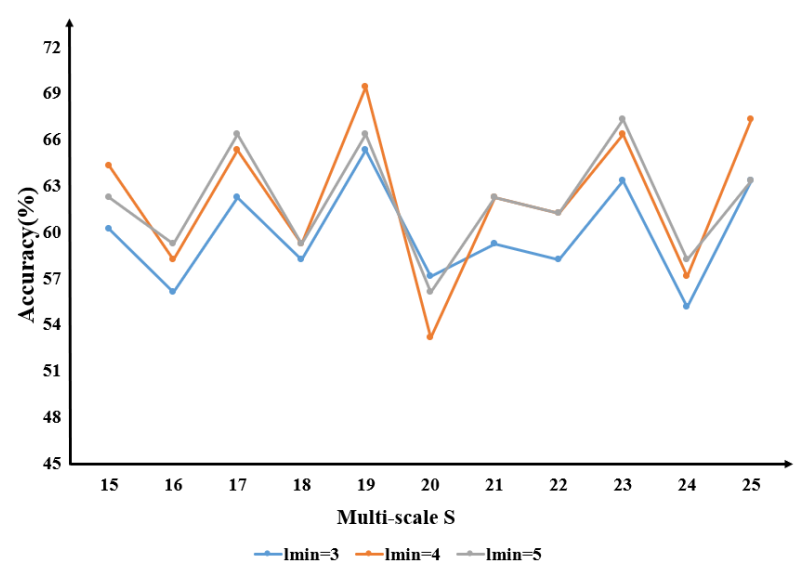

Fig. 4. Accuracy of different $1 \mathrm{~min}$ and multi-scale $\mathrm{S}$. In this study, $\mathrm{S}$ was set to $[15,25]$ and $\operatorname{lmin}$ was set to $3,4,5$. 


\section{Discussion}

The activity of the autonomic nervous system consisting of the sympathetic and parasympathetic nerves is generally believed to be reflected in the low and high frequency bands of heart rate variability [13]. LF/HF has been widely used to quantify the extent of sympathetic balance. As shown in Table 1, LF/HF can significantly distinguish the control and CHF samples $(p<0.001)$, which is consistent with other findings studying HRV features of CHF [14]. The effectiveness of this traditional indicator $\mathrm{LF} / \mathrm{HF}$ has been demonstrated.

Previous studies have shown that MSORP_DET can be applied to multi-time scale complexity analysis, reflecting the comparison of recurrence states and all possible states of the system as well as the aggregation degree of the phase space trajectories. Thus, MSORP_DET can be used to explore the nonlinear dynamic characteristics of electrical signals. Therefore, this study used MSORP_DET to evaluate the complexity of HRV in patients with CHF. The result showed that this index could also significantly distinguish the normal and CHF group $(p<0.001)$, which verified the feasibility of this new index for CHF classification and early stage CHF detection. Though the Acc, Sen, and Spe of MSORP_DET are lower than those of LF/HF, their combination obtained the highest screening Acc, and the Sen and Spe are more balanced. This shows that these two indices have their own advantages in CHF assessment. The frequency domain index LF/HF is very sensitive to the stationarity and periodicity of time series. At the same time, the actual HRV signal is not periodic, and its stationarity is often affected by the increase of signal length. Because MSORP_DET has a lower requirement in the stationarity and noise of the signal, it can make up for the shortcoming of the LF/HF. Therefore, combining these two indices is a suitable method to investigate HRV signals in CHF assessment.

In this study, MSORP_DET was used to analyze the HRV of normal and congestive heart failure patients. MSORP_DET can reflect the recurrence of a trajectory, the higher the MSORP_DET of a signal, the lower the complexity is, and the stronger the certainty (predictability) of the system. Figure 1 showed that the MSORP_DET of the CHF group was smaller than that of the normal group. It indicates that the probability of trajectories approaching each other in phase space of CHF patients was smaller, the similarity of time series was lower, and the short-term variability of the autonomic nervous system was more complex, changes in heart rate variability had occurred. This result is consistent with the past study's result that the rhythm changes of the autonomic nervous system were more complex and rapid in the CHF patients [15]. Compared with the CHF patients, the MSORP_DET of the normal group is higher, which indicates that the heart rate regulation system is deterministic and periodic. The autonomic nerve regulation ability is strong, which makes the heart rate fluctuation tend to be stable.

$\mathrm{HRV}$, as a life signal, is a typical long-term correlation signal with multi-scale. Therefore, the DET parameter was investigated on multiple time scale $S$, and the time scale with the highest Acc was selected. Figure 4 shows that at different time scales, the Acc fluctuates greatly and reaches the maximum value at $S=19$. It is thus clear that an appropriate time scale is helpful to highlight the structural differences of order recurrence plots in normal and pathological states.

The selection of embedding dimension $\mathrm{m}$, the delay time $\tau$, and the threshold value lmin is the key point. If $\mathrm{m}$ is too small, the corresponding order pattern is fewer, resulting in a larger DET; if $\mathrm{m}$ is too large, some information will be missed, resulting in a smaller DET. Therefore, in this study, $\mathrm{m}$ is set to 3 , and $\tau$ is set to 1 . When lmin is too small, too many diagonal lines will be included, the DET value will become larger; if $1 \mathrm{~min}$ is too large, it cannot contain all the diagonal structures in the recursive diagram, the DET value will become smaller. As shown in Figure 4, the fluctuation direction of three curves with $\operatorname{lmin}=3,4,5$ is generally the same. The Acc reached the highest when $\operatorname{lmin}=4$, and decreased obviously when $\operatorname{lmin}=3$, indicating that when $\operatorname{lmin}<=3$, too many diagonal structures in the recursive graph were contained.

This study has some limitations. First, the total number of samples used in the study was 98 , which is slightly insufficient. And the age difference between the two groups may affect the experimental results. Second, the samples did not have a severity level for congestive heart failure, so we could not differentiate the patients at multiple levels. Finally, due to the high complexity of the MSORP_DET algorithm, the algorithm's performance needs to be improved. It can only deal with short sequences [16] at present. In future studies, these limitations can be taken into consideration.

\section{Conclusion}

In this study, MSORP_DET was proposed as a new method to analyse $\overline{H R V}$ in patients with CHF. MSORP_DET is a nonlinear index that can significantly distinguish between the normal and CHF groups and show that the complexity of HRV in patients with CHF is higher than that in normal people. Hence, the MSORP_DET used in this experiment provides a new perspective for screening patients with CHF.

\section{References}

1. Figueroa, M.S. and J.I.J.R.c. Peters, Congestive heart failure: Diagnosis, pathophysiology, therapy, and implications for respiratory care. 2006. 51(4): p. 403412.

2. Schocken, D.D., et al., Prevalence and mortality rate of congestive heart failure in the United States. 1992. 20(2): p. 301-306.

3. Chen, W., et al., A Novel and Effective Method for Congestive Heart Failure Detection and Quantification Using Dynamic Heart Rate Variability Measurement. 2016. 11(11): p. e0165304.

4. Nolan, J., et al., Prospective Study of Heart Rate Variability and Mortality in Chronic Heart Failure 
Results of the United Kingdom Heart Failure Evaluation and Assessment of Risk Trial (UK-Heart). 1999. 98(15): p. 1510-1516.

5. Sanderson, J.E.J.H.F.R., Heart Rate Variability in Heart Failure. 1998. 2(4).

6. Billman, G.E.J.F.i.P., The $L F / H F$ ratio does not accurately measure cardiac sympatho-vagal balance. 2013. 4: p. 26.

7. Chen, M., et al., Empirical Mode Decomposition as a Novel Approach to Study Heart Rate Variability in Congestive Heart Failure Assessment. 2019. 21(12): p. 1169.

8. Eckmann, J.P., S.O. Kamphorst, and D.J.E.L. Ruelle, Recurrence Plots of Dynamical Systems.

9. 1987. 4(9): p. 973-977.

10. Zbilut, J.P. and C.L.J.P.L.A. Webber, Embeddings and delays as derived from quantification of recurrence plots. 1992. 171(3-4): p. 199-203.

11. Aparicio, T., et al., Detecting determinism using recurrence quantification analysis: Three test procedures. 2005. 65(3-4): p. 768-787.

12. Cysarz, D., et al., Symbolic transformations of heart rate variability preserve information about cardiac autonomic control. 2015. 36(4): p. 643-657.

13. Chen, et al., An Automatic Screening Approach for Obstructive Sleep Apnea Diagnosis Based on SingleLead Electrocardiogram. 2015.

14. Wilhelm, V.R., et al., Resolving Ambiguities in the LF/HF Ratio: LF-HF Scatter Plots for the Categorization of Mental and Physical Stress from HRV. 2017. 8: p. 360-.

15. Luo, D., et al., The interaction analysis between the sympathetic and parasympathetic systems in CHF by using transfer entropy method. 2018. 20(10).

16. Pan, W., et al., Multi-Frequency Components Entropy as Novel Heart Rate Variability Indices in Congestive Heart Failure Assessment. 2019: p. 1-1.

17. Shang, et al., Multiscale recurrence quantification analysis of Order recurrence plots. 2017. 